\title{
Proton-pump inhibitor-responsive esophageal eosinophilia
}

INSERM

\section{Source}

INSERM. (1999). Orphanet: an online rare disease and orphan drug data base. Protonpump inhibitor-responsive esophageal eosinophilia. ORPHA:411696

Proton-pump inhibitor-responsive esophageal eosinophilia (PPI-REE) is a rare, gastroenterologic disease characterized by typical clinical, endoscopic and histological features of eosinophilic oesophagitis (i.e. symptomatic oesophageal dysfunction associated with eosinophil-predominant mucose infiltrate) which completely remits upon proton pump inhibitor therapy. 